\section{Bromine-Doped Pentacene Acts as a Phototransistor with Gain over a Large Spectral Range}

Recent interest in the development of organic compounds for application as electronic devices has been stimulated in part by the capability of depositing the compounds over large areas on flexible substrates and at a low manufacturing cost. Doped-pentacene thin films have the potential to work as field-effect transistors (FETs) or photovoltaic diodes due to their high charge carrier mobility. Doping of pentacene enhances the benefits obtained from its electronic properties, and this represented an advantage for Jan Hendrik Schön and Christian Kloc from Bell Laboratories/Lucent Technologies when they fabricated an optically activated FET, as they reported in the May 28 issue of Applied Physics Letters. The researchers obtained such a device by using bromine-doped pentacene, which becomes a $p$-type semiconductor.

Single crystals of pentacene, $1-\mu \mathrm{m}$ thick, were grown from vapor in a flow of hydrogen. The source and drain contacts were 300-nm-thick gold strips deposited by shadow masking, and the Schottky gate electrode was a 30-nm-thick aluminum strip. A Xe lamp and a monochromator or a He-Ne laser was used to illuminate the device. As the device is normally off, it develops a potential barrier at the AlSchottky contact. In this case, the drain current increases with increasing gate bias and saturates with increasing drain voltage. The operative conditions of this device can be manipulated by changing the dopant amount, film thickness, or Schottky barrier height.

Illumination changes the behavior of the drain current and causes the device response to increase abruptly when the energy of the light quanta are equivalent

\section{$\mathrm{MgB}_{2}$ Superconductors Update Available}

The discovery of $39 \mathrm{~K}$ superconductivity in crystallographically simple $\mathrm{MgB}_{2}$ in January has launched a flurry of intense international research and development activities, as scientists search for higher transition temperatures $\left(T_{\mathrm{c}}\right)$ as well as better understanding and improvement of $\mathrm{MgB}_{2}$ 's materials characteristics. See the Materials Research Society Web site for an update on recent findings presented at the 2001 MRS Spring Meeting: www.mrs.org/meetings/ spring2001/news/mgb2.html. to that of the absorption edge of the semiconductor. This device seems to act in a similar manner to a photodiode which, however, incorporates the amplification of a FET. Changes in the illumination intensity affect the voltage at the gate and therefore affect the drain current, whose square root was found to vary logarithmically with illumination power. For this device, a constant gain of 8 can be achieved in the energy range between $1.8 \mathrm{eV}$ and $2.5 \mathrm{eV}$. The authors define gain as the ratio of the total external current to the incident photon flux. In recent experiments, the researchers also observed a similar behavior in iodine-doped pentacene single crystals and thin films.

SIARI S. SOSA

\section{Self-Assembled DNA Basis Serves as Semiconductor in MSM Photodetector}

R. Rinaldi and colleagues at Università di Lecce (National Nanotechnology Laboratory of INFM) and Università di Bologna in Italy have fabricated metalsemiconductor-metal (MSM) photodetectors using a layer of a DNA basis. By varying the concentrations of a chloroform solution of this DNA basis-a deoxyguanosine (DG) derivative-the researchers were able to find a concentration that would self-assemble once deposited on a silicon dioxide substrate. The selfassembly took place in an approximately 120-nm-wide space between 5-mm-long gold electrodes. Continued pursuits with these materials may lead to extremely dense logic and memory circuits.

As reported in the May 21, 2001 issue of Applied Physics Letters, the researchers used electron-beam lithography to pattern $\mathrm{a} \mathrm{SiO}_{2}$ substrate. Next, they used liftoff processing and layers of chromium and gold to complete the 10- $\mu$ m-wide electrodes. After completing the electrodes, they used a Hamilton syringe to drop a solution of deoxyguanosine derivative in chloroform between the electrodes on the substrate. When the solvent evaporated, a biological semiconductor material with an energy bandgap of approximately $3.4 \mathrm{eV}$ self-assembled. By superimposing the chemical scheme onto atomic force microscopy images, the researchers demonstrated that the DG had self-assembled into aligned biological wires. However, based on $I-V$ characteristics, the researchers believe that charge transport does not occur directly through these wires. The researchers said that, instead, "conductivity is a bandlike phenomenon involving the crystal of DG as a whole." They said that the effect of the internal dipole induces the electron donor properties of the DG crystal.

The researchers anticipate further development of molecular-scale electronics as a result of the high responsivity $(1 \mathrm{~A} / \mathrm{W})$ of this biological material. The molecular scale of this photodetector tempts the researchers with the possibility of biomolecular planar devices written by inkjet printers.

PAMELA JOHNSON

\section{Synthetic Clay Removes Radium from Water and Soil}

Radium, a natural decay product of uranium, is found where large deposits of uranium are mined, but is also present in many other areas in rocks and soils. Coal and phosphate processing also produce tailings that contain radium. Having tested a variety of synthetic micas for radium removal, Sridhar Komarneni, professor of clay mineralogy at The Pennsylvania State University, has found that sodium- 4 mica serves as a prime synthetic clay for this purpose.

Natural mica is a mineral containing a combination of aluminum, silica, magnesium, and potassium. The mineral is found in sheets that are bonded to each other to form a solid, layered mass. Komarneni said that natural mica has a closed structure with all the spaces between layers filled and is not a good ion-exchange medium.

Sodium-4 mica, like natural mica, contains aluminum, silica, and magnesium, but each potassium atom is replaced by two sodium ions, and fluorine is also added. The two sodium ions take up more space than the potassium ion and the layers of mica become offset, creating a space to capture water and radium.

Komarneni said, "Sodium-4 mica has an interlayer spacing of $2.6 \AA$, too small to capture ions of hydrated sodium, calcium, magnesium, or potassium. Radium, however, is less hydrated and therefore small enough to fit between the layers, as are barium, copper, nickel, and zinc."

These other transition metals are not usually found in great abundance in radium-contaminated water or in tailings containing radium, so they would not compete for space between the layers. As Komarneni reported in the April 12 issue of Nature, when the mica is filled with radium, a shift in the layers occurs and the atoms of radium are trapped between the layered structures.

"Once the radium is trapped, it will not leave the mica," Komarneni said. "Disposal and storage requirements would then depend only on the radioactivity of the material and not whether radium could 
leach out of the clay. Very low level radioactive clay could simply be buried."

Komarneni and co-researchers Naofumi Kozai, a visiting scientist from the Japan Atomic Energy Research Institute, and William J. Paulus, master's degree recipient, now at General Motors Corporation, found that if the mica is only partially filled with radium at the time of disposal, then heating to above $100^{\circ} \mathrm{C}$ will lock the radium in place.

Sodium -4 mica, the researchers found, is easily synthesized by heating kaolinitea naturally occurring clay with an equal ratio of silicon and aluminum-with magnesium oxide and sodium fluoride to about $815^{\circ} \mathrm{C}$.

Sodium- 4 mica could be used in conventional ion-exchange columns to remove radium from water, but would first need to be pelletized. To immobilize radium from mine or mill tailings, the researchers said that mixing the clay with the tailings is sufficient. They also suggested that the clay could be used to line ponds that receive radium-containing tailing water to prevent migration from the pond, or clay curtains can be placed around tailings to keep the radium inside.

\section{Linear Defects Stabilize Magnetic Domain Walls}

Ultrathin cobalt films or multilayered structures, magnetized perpendicular to the thin-film planes, have greatly increased storage densities by solving the thermal "flips" (i.e., magnetic spin reversals) problem that leads to the loss of stored information. However, extension of this technique to storage densities larger than 1 Tbit in. $^{-2}$ suffers from the roughness and mobility of the magnetic domain walls (DWs), which prohibits closer packing of the storage bits. In order to overcome this problem and achieve even higher storage density, researchers from Los Alamos National Laboratory and IBM T.J. Watson and Almaden Research Centers have utilized long-range strain fields caused by introduced linear defects.

When ultrathin trilayers (sandwich structures $)$ of $\operatorname{Pt}(3 \mathrm{~nm}$, top $) / \mathrm{Co}(0.7$ $\mathrm{nm}) / \mathrm{Pt}(2 \mathrm{~nm}$, bottom) are deposited, the domains exist in round shape with rugged walls. The same problem occurs in pattern nucleation sites. The velocity of DWs increases swiftly when the magnetic field is beyond $H_{\mathrm{c}}=750$ Oe (the velocity

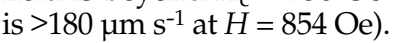

Noting examples that column defects can strongly localize wandering vortex lines in high-temperature superconductors, the researchers installed a line defect in the cobalt layer by clamping the substrate to produce anisotropic tension during the cobalt deposition, and then releasing the sample. As reported in the March 22 issue of Nature, the introduced $y$-axis-invariant strain field $\varepsilon(x)$ influences the behavior of domains through magnetoelastic coupling. First, it accommodates the walls along the linear defect. Second, by increasing the elasticity, it reduces the wall roughness. Finally, it decelerates the motion of DWs to a nearly full stop when the field is high enough.

Polar magneto-optic Kerr microscopy images show that the conformance of the DWs to the defect line occurs even at a large distance $(\sim 300 \mu \mathrm{m})$. The closer the DWs come to the defects, the smoother (straighter) they are. The deceleration rate also depends on the proximity to the defects. The researchers found a nonlinear relationship of $v-H$ curve (where $v-H$ refers to voltage-versus-field), which is regarded as a sign of glassy (creep) dynamics. A higher field $H$ is needed to get closer to the line defects, but after a

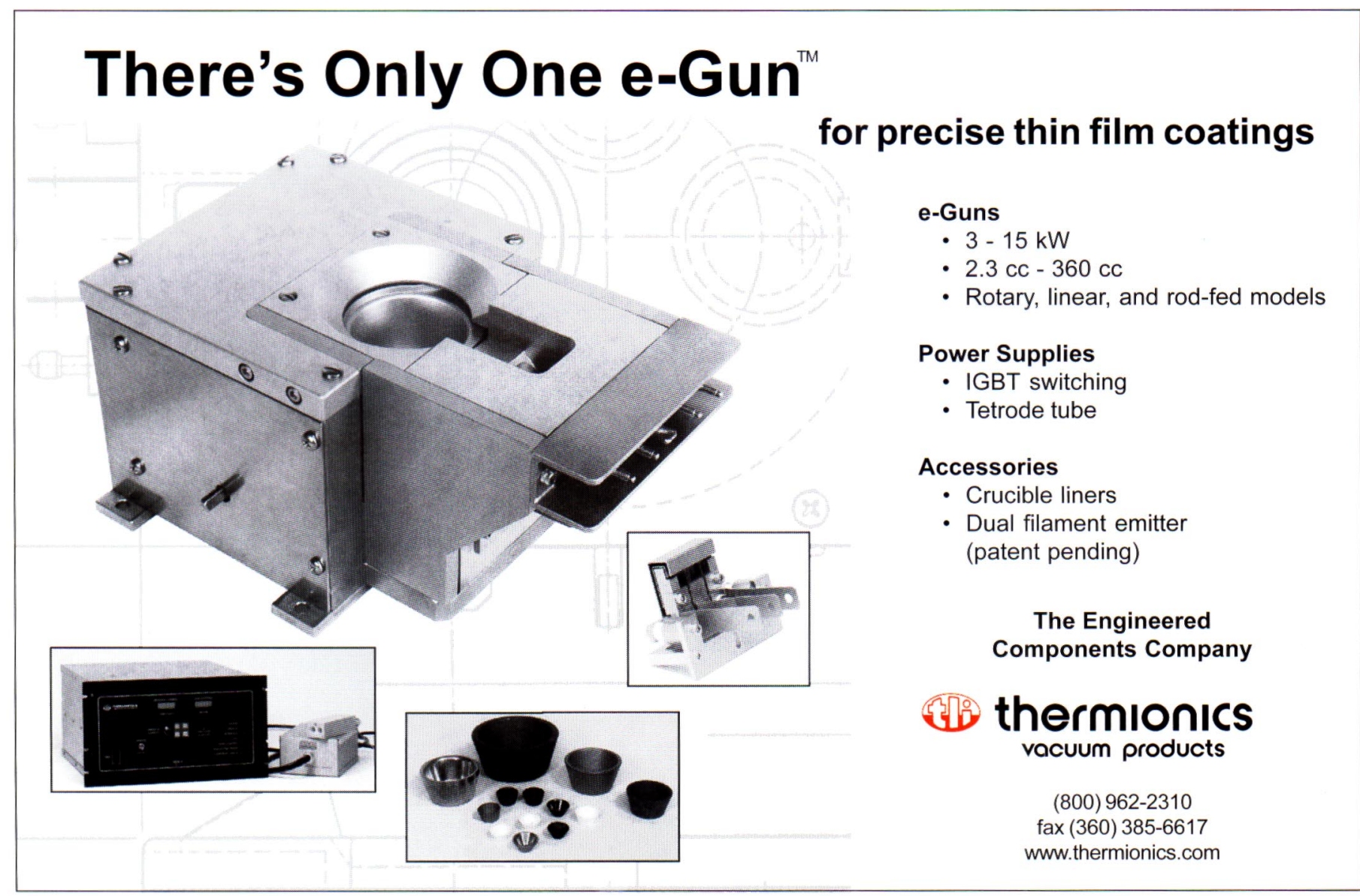

Circle No. 5 on Inside Back Cover 